\title{
Neoantigen quality, not quantity
}

\section{Nicholas McGranahan 1,2 and Charles Swanton 1,3}

1. Cancer Research UK Lung Cancer Centre of Excellence, University College London Cancer Institute, Paul O'Gorman Building, 72 Huntley Street, London, WC1E 6BT, United Kingdom

2. Cancer Genome Evolution Research Group, University College London Cancer Institute, University College London, London, WC1E 6BT, UK

3. Cancer Evolution and Genome Instability Laboratory, The Francis Crick Institute, 1 Midland Rd, London, NW1 1AT, United Kingdom

\begin{abstract}
Prioritizing expressed clonal neoantigens in genes required for cancer cell survival may reduce the likelihood of resistance to neoantigen therapies.
\end{abstract}

\section{Introduction}

Intratumor heterogeneity poses a major problem to the management of early and advanced cancers. Increasing evidence has supported the targeting of clonal events present in all tumor cells at all sites of metastatic disease. Indeed, therapies targeting cancer founder events, present in all tumor cells, have proven effective for a limited time; for example, 3rd generation epidermal growth factor receptor (EGFR) tyrosine kinase inhibitors (TKIs), used in the management of patients with advanced non-small cell lung cancers (NSCLCs) with EGFR activating mutations, result in a median progression-free survival of approximately 19 months (1). However, in the metastatic setting, the sobering reality is that resistance is the rule, not the exception, with the majority of patients succumbing to their disease. Furthermore, late-stage, treatment-refractory disease may be characterized by a myriad of subclonal somatic copy number events and point mutations that render tumors inexorably refractory to therapy.

The rapidly evolving tumor somatic mutational landscape must be grappled with. Some drug resistance events in more genomically simple (often non-carcinogen-induced) tumors are predictable and themselves actionable. For example, the third generation EGFR TKI, osimertinib, has proven remarkably effective in the management of NSCLCs which harbor T790M EGFR resistance mutations, which render them resistant to first generation TKIs (1). However, the majority of NSCLCs are characterized by extensive genomic complexity and ongoing chromosomal instability, in part resulting from the mutagenic insults of carcinogen exposure. Therefore, conventional pharmaceutical small molecule approaches targeting single oncogenic drivers are unlikely to be able to compete with the multitude of evolutionary resistance mechanisms effectively.

Over the last decade, cancer-specific neoantigens, deriving from somatic mutations leading to changes in protein sequences, have emerged as one of the major targets of CD8+ T cells in the immune microenvironment. These $\mathrm{T}$ cells can be potentiated by immune checkpoint inhibitors, which enhance CD8 function or deplete T regulatory cells.

Tumor mutational burden and predicted neoantigen load correlate with response to checkpoint inhibitor blockade in NSCLC (2). Indeed, for a limited subset of patients, checkpoint inhibitor therapy (CPI) provides hope of long-term disease control. However, 
acquired resistance to CPI, as defined by the Response Evaluation Criteria in Solid Tumors (RECIST), is emerging as a major clinical problem. Intriguingly, where data exist, acquired resistance is less frequent and not inevitable in some tumor types, unlike with targeted therapies. For example, in melanoma, only about $1 / 4$ to $1 / 3$ of patients with metastatic melanoma whose tumors respond to anti-programmed cell death protein-1 (anti-PD-1) or anti-cytotoxic T-lymphocyte-associated protein 4 (anti-CTLA4) therapy will develop acquired resistance (3).

\section{Identifying key features of neoantigens}

Tumor mutational burden and PD-L1 expression are imperfect biomarkers that can be used to help stratify patients who derive clinical benefit from CPI therapy. But what distinguishes a tumor with profound sensitivity to checkpoint inhibitor therapy from one that is initially sensitive but eventually recurs? Although there are likely a myriad of different mechanisms of resistance to immunotherapy, if we assume that the final common pathway of robust anti-tumor activity after CPI therapy is directed, in part, against the tumor neoantigen, it is worth considering the qualitative features of neoantigens that lead to a robust and durable response.

\section{Clonal fraction}

Clonal neoantigens are emerging as a focus of immune-mediated control (Fig. 1). Indeed, the clonality of a neoantigen is likely important for a number of reasons. First, recent evidence from mouse modeling suggests that a minimum cell fraction may be required to elicit $\mathrm{T}$ cellmediated immune rejection (4); clonal neoantigens by definition are present at a higher cancer cell fraction than subclonal neoantigens. Second, subclonal neoantigens are not presented by every cancer cell and so are potentially less likely to result in effective immune control across all sites of disease. Third, in some tumor types such as NSCLC, where whole genome doubling events occur in over $70 \%$ of patients as an early clonal event, neoantigens occurring before genome doubling will be at twice the allelic fraction compared to a clonal neoantigen occurring after genome doubling, thereby potentially enhancing $\mathrm{T}$ cell recognition. The importance of clonal neoantigens is supported by evidence that the burden and fraction of clonal neoantigens correlates with response to checkpoint inhibitor therapy (5).

\section{Similarity to self or known antigens}

The immune system is poised to recognize non-self. Thus, the degree to which a peptide is non-self may influence the likelihood of effective immune control. Recent work suggests that frameshift insertion mutations, driving new open reading frames, or "neo-ORFs" encoding multiple (>10) new amino acids may be a particularly potent source of neoantigen $(6)$. It remains unclear whether sequence homology to known antigens can improve ability to identify neoantigens that predict response to CPI. Thus, certain mutational processes, such as mismatch repair deficiency, may potentially result in more potent neoantigens than, for example, spontaneous deamination of methylated cytosines.

\section{Expression}

If a mutation is never transcribed or translated, it cannot stimulate an immune response (Fig. 1). Evidence for purifying selection of neoantigens residing in genes which are normally expressed in lung cancer highlights the importance of considering expression as a key feature of identifying bona fide neoantigens (7). Moreover, even for putative neoantigens occurring in genes which would normally be expected to be expressed, there is evidence for transcriptional repression. Promoter methylation of predicted high-affinity binding neoantigens was seen in approximately $20 \%$ of non-expressed neoantigens in the TRACERx study of untreated early stage NSCLC (7). Other mechanisms of transcript down-regulation, including exon skipping 
and chromatin remodeling, may therefore also represent avenues of immune escape and should be considered when identifying likely neoantigenic mutations.

\section{Human Leukocyte Antigen (HLA) binding}

The HLA complex encodes genes responsible for antigen presentation and is highly polymorphic. Recent work suggests that both germline HLA homozygosity and the presence of certain alleles may influence the likelihood of CPI benefit, at least in certain cancer types (8). During cancer evolution, HLA loss is a well-documented mechanism of immune evasion. In NSCLC, clonal or subclonal HLA loss of heterozygosity (LOH) occurs in approximately $40 \%$ of patients and is enriched in immune hot microenvironments (7). Emerging subclones would be predicted to evade the host immune response. Consistent with this hypothesis, HLA $\mathrm{LOH}$ is associated with an expansion of mutations in emerging subclones, and is likely to be permissive for ongoing branched evolution. Beyond HLA loss, mutations or DNA copy loss to other components of the neoantigen presentation machinery is emerging as a common feature of NSCLC, with over $75 \%$ of lung squamous and $50 \%$ of lung adenocarcinoma tumors harboring at least one disruption to this pathway (7). HLA loss may also act as a resistance mechanism to targeted tumor-infiltrating lymphocyte treatment, highlighting the need to consider which allele a putative neoantigen may bind to. In a seminal study of T cell therapy, a patient with metastatic colorectal cancer was treated with four distinct $\mathrm{T}$ cell clones recognizing KRAS G12D in an HLA-C*802 restricted manner (9). 9 months after therapy, one lesion progressed and was found to have lost the HLA-C*802 allele, leaving the tumor with a HLA-C*1601 allele. If the patient had been germline homozygous at the HLA-C*802 locus, this might have restricted the evolution of resistance to KRAS G12D directed T cell therapy. Prioritizing neoantigens that bind multiple HLA molecules may also limit the evolution of resistance to neoantigen-directed therapies driven by HLA loss.

\section{Likelihood of neoantigen loss}

Because most putative neoantigens are generally considered passenger rather than driver events, their loss through chromosomal instability during tumor evolution may be readily tolerated. Anagnostou and colleagues (10) revealed how clonal neoantigens can be lost at progression after CPI therapy, likely as a mechanism of resistance. Similarly, in immune cold, untreated early-stage NSCLC, evidence of subclonal loss of previously clonal neoantigens can be found, indicating immune selection. These data raise questions over the challenges of turning immune cold tumors into immune hot tumors; conceivably, the immune cold phenotype may reflect, in part, the loss of clonal neoantigens during tumor evolution. Therapies directed at restoring immune cell infiltration may be hindered by the absence of robust neoantigens.

Given that certain areas of the genome are seldom or never subject to copy number loss, the local genomic position of putative neoantigens may represent an important feature to consider in defining the likelihood of eliciting an immune response. When mutations that do not disrupt gene function occur in genes required for tumor cell survival (housekeeping or viability genes) and are recognized as neoantigens, there may be a profound fitness cost if these genes are deleted through copy number loss or transcriptionally repressed through methylation. Such neoantigens could be considered as essential neoantigen events as opposed to neoantigen passengers, whose deletion or repression results in no fitness impact to the cancer subclone. Essential neoantigens could occur in cancer driver genes, but also in genes required for cancer cell viability. Neoantigens in cell viability genes, in haploid regions of loss of heterozygosity that would prevent further disruption to the remaining allele through deletion or transcriptional repression might be particularly potent sources of immune control (Fig. 1). 


\section{Summary}

Evidence is building for ongoing tensions between the adaptive immune system and evolving tumors. On one hand, mutations provide fitness through the activation of key driver events or loss of tumor suppressor genes during evolution. On the other, a minority of mutations may result in neoantigens and provide beacons to alert the immune system to an evolving tumor. Exploring the differences in the neoantigen repertoires between patients who have prolonged benefit compared to those whose tumors develop acquired resistance to immunotherapy may shed further light on the selection of optimal neoantigens for therapy. The reasons some tumors respond potently to immunotherapy may reflect both the quantity and the quality of their neoantigens. High-quality neoantigens might include expressed clonal neoantigens in essential genes which bind to multiple HLA alleles and cannot be repressed or deleted by virtue of their genomic position. Conceivably, such neoantigen events could act as potent sources of immune surveillance, vaccine therapy, and adoptive T cell therapy.

\section{Figure Legends}

Figure 1. Prioritising quality neoantigens

\section{References and notes}

1. A. Murtuza et al., Novel Third-Generation EGFR Tyrosine Kinase Inhibitors and Strategies to Overcome Therapeutic Resistance in Lung Cancer. Cancer Res 79, 689698 (2019).

2. N. A. Rizvi et al., Cancer immunology. Mutational landscape determines sensitivity to PD-1 blockade in non-small cell lung cancer. Science 348, 124-128 (2015).

3. P. Sharma, S. Hu-Lieskovan, J. A. Wargo, A. Ribas, Primary, Adaptive, and Acquired Resistance to Cancer Immunotherapy. Cell 168, 707-723 (2017).

4. R. S. Gejman et al., Rejection of immunogenic tumor clones is limited by clonal fraction. Elife 7, (2018).

5. N. McGranahan et al., Clonal neoantigens elicit T cell immunoreactivity and sensitivity to immune checkpoint blockade. Science 351, 1463-1469 (2016).

6. S. Turajlic et al., Insertion-and-deletion-derived tumour-specific neoantigens and the immunogenic phenotype: a pan-cancer analysis. The lancet oncology 18, 1009-1021 (2017).

7. R. Rosenthal et al., Neoantigen directed immune escape in lung cancer evolution

. Nature Mar;567(7749):479-485, (2019).

8. D. Chowell et al., Patient HLA class I genotype influences cancer response to checkpoint blockade immunotherapy. Science 359, 582-587 (2018).

9. E. Tran, P. M. Robbins, Y.-C. Lu, T. D. Prickett, e. al., T-Cell Transfer Therapy Targeting Mutant KRAS in Cancer. New England Journal of Medicine 375, (2016).

10. V. Anagnostou et al., Evolution of Neoantigen Landscape during Immune Checkpoint Blockade in Non-Small Cell Lung Cancer. Cancer discovery 7, 264-276 (2017).

\section{Funding}

N.M is a Sir Henry Dale Fellow, jointly funded by the Wellcome Trust and the Royal Society (Grant Number 211179/Z/18/Z), and also receives funding from Cancer Research UK, Rosetrees, and the NIHR BRC at University College London Hospitals, and the CRUK University College London Experimental Cancer Medicine Centre. C.S. is Royal Society Napier Research Professor. This work was supported by the Francis Crick Institute that 
receives its core funding from Cancer Research UK (FC001169, FC001202), the UK Medical Research Council (FC001169, FC001202), and the Wellcome Trust (FC001169, FC001202). C.S. is funded by Cancer Research UK (TRACERx and CRUK Cancer Immunotherapy Catalyst Network), the CRUK Lung Cancer Centre of Excellence, Stand Up 2 Cancer (SU2C), the Rosetrees Trust, Butterfield and Stoneygate Trusts, NovoNordisk Foundation (ID16584), the Prostate Cancer Foundation, the Breast Cancer Research Foundation (BCRF). The research leading to these results has received funding from the European Research Council (ERC) under the European Union's Seventh Framework Programme (FP7/20072013) Consolidator Grant (FP7-THESEUS-617844), European Commission ITN (FP7PloidyNet 607722), ERC Advanced Grant (PROTEUS) has received funding from the European Research Council under the European Union's Horizon 2020 research and innovation programme (grant agreement No. 835297), Chromavision - this project has received funding from the European's Union Horizon 2020 research and innovation programme (grant agreement No. 665233). Support was also provided to C.S. by the National Institute for Health Research, the University College London Hospitals Biomedical Research Centre, and the Cancer Research UK University College London Experimental Cancer Medicine Centre.

\section{Competing interests:}

N.M. has stock options in and has consulted for Achilles Therapeutics. CS has received grant or research support from Pfizer, AstraZeneca, BMS, Ventana and NovoNordisk. CS has received honoraria for consultancy or scientific advisory boards from Boehringer Ingelheim, Eli Lily, Servier, Novartis, Roche-Genentech, GlaxoSmithKline, Pfizer, BMS, Celgene, AstraZeneca, Illumina, Sarah Cannon Research Institute. CS has stock or stock options in Apogen Biotechnologies, Epic Bioscience, GRAIL, and Achilles Therapeutics. CS and NM have patents pending relating to targeting neoantigens (PCT/EP2016/059401); identifying patent response to immune checkpoint blockade (PCT/EP2016/071471); determining HLA LOH (PCT/GB2018/052004) and predicting survival rates of cancer patients (1901439.8). In addition, CS has patents pending related to treating cancer by targeting Insertion/deletion mutations (PCT/GB2018/051893); identifying insertion/deletion mutation targets (PCT/GB2018/051892); stratifying renal cell carcinoma patients (1805876.8); detecting tumor recurrence (PCT/GB2017/053289); detecting tumor mutations (PCT/US2017/28013); identifying responders to cancer treatment (PCT/GB2018/051912). 